\title{
Cambios en la cinemática articular tras entrenamiento de la marcha con exoesqueleto robótico ambulatorio
}

\author{
del-Ama, Antonio J. ${ }^{\text {a }}$, Megía, Álvaro ${ }^{a}$; Lozano-Berrio, Vicente ${ }^{a}$; Gil-Agudo, Ángel ${ }^{\text {b }}$ \\ ${ }^{a}$ Unidad de Biomecánica y Ayudas Técnicas. Hospital Nacional de Parapléjicos -Finca La Peraleda \\ S/N, Toledo, España. ajdela@sescam.jccm.es, \\ ${ }^{\mathrm{b}}$ Servicio de Rehabilitación. Hospital Nacional de Parapléjicos -Finca La Peraleda S/N, Toledo, \\ España.
}

\begin{abstract}
Ambulatory robotic exoskeletons for intensive gait training have been proposed as alternate to improve patient involvement. In this article, a case study is presented in which the effect of exoskeleton-assisted gait training on lower limb joint kinematics during 4 training sessions is assessed. The results suggest changes in joint kinematics during exoskeleton walking training sessions. These changes tend to be reduced as the training sessions increase, although it has not been possible to demonstrate statistically. The experimental procedure can be considered valid, this study being the basis for a broader analysis with a greater number of subjects and training sessions.
\end{abstract}

Keywords: gait, biomechanics, Robotics, exoskeletons, sensors

\section{Resumen}

Los exoesqueletos robóticos ambulatorios para el entrenamiento intensivo de la marcha, asegurando la involucración del paciente en la tarea. En este artículo se presenta un estudio piloto sobre el efecto del entrenamiento de la marcha asistida con exoesqueleto sobre la cinemática de las articulaciones de la extremidad inferior de un único paciente durante 4 sesiones de entrenamiento con exoesqueleto ambulatorio. Los resultados sugieren cambios en la cinemática articular durante las sesiones de entrenamiento de la marcha con exoesqueleto. Los cambios tienden a reducirse según aumentan las sesiones de entrenamiento, aunque no se ha podido demostrar estadísticamente. El procedimiento experimental puede considerarse válido, siendo este estudio la base para un análisis más amplio con un mayor número de sujetos y sesiones de entrenamiento.

Palabras clave: marcha, biomecánica, robótica, exoesqueletos, sensores inerciales. 


\section{Introducción}

La afectación de la función de marcha tras una lesión medular es una de las principales secuelas tras la lesión medular. Se estima que aproximadamente la mitad de las plesiones medulares son incompletas, cuya afectación de las extremidades es variable [1]. La rehabilitación de la capacidad de marcha es uno de los objetivos principales de las personas con lesión medular [2]

Actualmente la reeducación de la marcha se fundamenta en los principios de la promoción de mecanismos neuroplásticos mediante la ejecución de tareas motoras de manera repetitiva e intensiva. La premisa es proporcionar al sistema nervioso dañado la información sensorial adecuada para estimular las redes neuronales intactas de la médula espinal para facilitar su activación, incluso cuando la parte supra-espinal está comprometida. Los circuitos neuronales responden a las activaciones sensoriales, modulando el movimiento, pudiendo inducir modificaciones permanentes con la repetición [19]. La repetición intensiva del movimiento de la marcha, descargando parcialmente el peso del paciente, ha sido el principal paradigma de entrenamiento que sigue estos principios [20]. Debido a la demanda física que este entrenamiento impone a los fisioterapeutas, a principios de los años 200 se desarrolló un robot capaz de realizar la movilización de las extremidades inferiores sobre un tapiz rodante con descarga parcial de peso, denominado Lokomat [21] La utilización de Lokomat permite incrementar la intensidad y duración del entrenamiento, si bien este incremento no se ha correspondido con un incremento en los resultados funcionales obtenidos por los grupos de pacientes [8], [10]. Hay algunos autores que sugieren que los motivos de esta aparente falta de eficacia residen en que este tipo de robots eliminan la variabilidad intrínseca de la marcha, un mecanismo conocido del aprendizaje motor, así como la involucración del paciente en la tarea [12]

Para mejorar las condiciones de entrenamiento de la marcha, los exoesqueletos robóticos ambulatorios constituyen una alternativa que permite asimismo el entrenamiento intensivo de la marcha, asegurando la involucración del paciente en la tarea, a cambio de un mayor coste metabólico y la consecuente reducción en el tiempo de tratamiento [22], [23]. Si bien existe evidencia sobre su seguridad y tolerancia, actualmente se está investigando su efecto terapéutico para la rehabilitación de la marcha [24]-[26]. Dada la similitud con los entrenadores robóticos de la marcha, se ha asumido que los mecanismos terapéuticos son los mismos en ambos sistemas, si bien los mecanismos biomecánicos y fisiológicos de la marcha ambulatoria asistida por robot no son exactamente los mismos [27]. Es necesario por tanto investigar los mecanismos de adaptación entre el usuario y el exoesqueleto, el efecto sobre los sistemas nerviosos central y periférico, las estrategias de compensación 
generadas por el usuario en respuesta a la asistencia y el impacto físiológico del entrenamiento de la marcha asistida por exoesqueleto.

En este artículo se presenta un estudio piloto sobre el efecto del entrenamiento de la marcha asistida con exoesqueleto sobre la cinemática de las articulaciones de la extremidad inferior. La hipótesis principal es que el entrenamiento de la marcha por un tiempo de 15 minutos provoca cambios inmediatos en la cinemática articular. Para ello, se plantea como objetivo analizar la cinemática de las articulaciones de la cadera, rodilla tobillo inmediatamente antes y después del entrenamiento de la marcha con exoesqueleto ambulatorio.

\section{Material y métodos.}

\subsection{Paciente: patología y afectación de la marcha.}

La paciente es una mujer de 51 años de edad con síndrome de lesión medular L1 ASIA C. Al inicio del programa de entrenamiento de la marcha con exoesqueleto presenta un índice muscular del miembro inferior de 16/14 (izquierda/derecha) sobre 25, espasticidad Ashworth $=0$ y $P E N=1$. La valoración funcional de la marcha responde a los siguientes valores: Test de los 10 metros $=36$ segundos; test de los 6 minutos $=99$ metros; Timed-upand-go $=43$ segundos; WISCI-II = 9; SCIM-III = 71/100.

La paciente deambula con andador sin supervisión con órtesis de tobillo en pie derecho para interiores. Usa silla de ruedas con propulsión manual para espacios exteriores.

\subsection{Exoesqueleto HANK}

El exoesqueleto HANK (Gogoa Mobility, Traña-Matiena, Bizkaia) Su diseño se basa en una estructura ortésica con una única barra lateral de soporte, con 3 grados de libertad ubicados en el plano lateral de la barra, correspondiendo con los actuadores para las caderas, rodillas y tobillos. Los actuadores están compuestos por motores de corriente continua sin escobillas y reductores Harmonic Drive, con relación de transmisión 160:1, encóders magnéticos y sensores de fuerza. Los actuadores integran una placa con la electrónica de control de los motores, la adquisición y tratamiento de las señales de los sensores, así como la comunicación con el controlador principal.

La arquitectura de control del exoesqueleto se basa en un microcontrolador que permite la comunicación mediante Bluethooth con la interfaz de control externa. Integra también un bus de comunicación CAN para mandar las consignas de control a los actuadores, así como recibir la información de los sensores. 
La interfaz de control se implementa mediante una aplicación instalada en una tablet o teléfono móvil con sistema Android. Permite varias acciones y modos de control. Para las sesiones de entrenamiento se han utilizado las siguientes: levantar, marcha continua, cambio de velocidad de la marcha, detención, y sentado.

\subsection{Sensores inerciales}

Para el registro del movimiento articular se utilizó un conjunto de sensores inerciales de TechnAid (Arganda del Rey, Madrid), compuestos por acelerómetro, giróscopo y magnetrómetro dispuestos ortogonalmente. Mediante un algoritmo de fusión sensorial basado en un filtro de Kalman modificado, los sensores estiman su orientación en el espacio respecto a un sistema de referencia no inercial constituido por la gravedad y el norte magnético terrestre. La comunicación entre los sensores se realiza mediante un bus SPI que transmiten la información a un concentrador de datos que a su vez permite la transmisión de datos mediante Bluethoot a un ordenador externo.

\subsection{Protocolo experimental.}

\subsubsection{Registro de la cinemática de la marcha}

Previamente a realizar el entrenamiento, se colocaron 4 sensores inerciales colocados en el pie, tibia, muslo y pelvis de la paciente, utilizando los soportes y cinchas del fabricante. Este montaje permite el cálculo de los ángulos articulares del tobillo, rodilla y cadera. Tras la puesta de los sensores, se realizó una calibración de la orientación de los sensores respecto a los principales ejes anatómicos de los segmentos en los que se ubican los sensores, pidiendo a la paciente que mantenga una posición erguida con las articulaciones alineadas, durante 3 segundos, y registrando la orientación de los sensores. Tras este procedimiento, se pide a la paciente que comience a andar en línea recta, utilizando para ello un andador. Se realiza la captura de los datos durante al menos 2 minutos. Tras la captura, la paciente pasa a una silla, donde se quitan los sensores y se pone el exoesqueleto.

\subsubsection{Entrenamiento de la marcha con exoesqueleto}

El exoesqueleto se alinea con las articulaciones de la paciente y se ajustan las cinchas hasta conseguir una sujeción firme y no molesta. Se pasa a bipedestación con la asistencia del exoesqueleto y el andador de la paciente. Tras inspeccionar la alineación del exoesqueleto con las articulaciones de la paciente, y verificar las cinchas, se comienza con el entrenamiento de la marcha. 
El entrenamiento de la marcha consiste en la realización de 15 minutos de marcha asistida por el exoesqueleto en línea recta y ajustando la velocidad de la marcha a la capacidad de la paciente, bajo criterio del terapeuta responsable del entrenamiento.

Tras los 15 minutos de marcha, se retira el exoesqueleto y se vuelven a colocar los sensores inerciales, y la paciente vuelve a realizar el procedimiento descrito anteriormente. El tiempo entre la finalización del entrenamiento de la marcha y el registro de la cinemática de la marcha es inferior a 5 minutos.

\subsection{Análisis de datos}

Los datos de los sensores inerciales se descargaron y trataron mediante MATLAB. En primer lugar se segmentaron los datos mediante un algoritmo especialmente diseñado para este experimento, detectando el contacto inicial a través de la verificación de los siguientes eventos: extensión completa de la rodilla, cadera en extensión y tobillo en flexión dorsal. Además, el algoritmo permite realizar una inspección visual del resultado de la detección, facilitando eliminar detecciones incorrectas.

Tras la detección se extrajeron las trayectorias articulares en el plano sagital de cada paso y se normalizaron entre 0 y $100 \%$. Para cada paso y articulación se obtuvieron obtienen las siguientes variables: máximo, mínimo, rango articular, y valor cuadrático medio. Además, para cada articulación y grado de libertad se obtuvo la curva resultante de promediar todos los pasos.

Se realizó una prueba t de Student para evaluar las diferencias entre las variables obtenidas previamente y tras cada entrenamiento de la marcha, así como entre el registro inicial del primer y último entrenamiento de la marcha. El valor de significación se estableció en $\mathrm{p}<0,05$.

\section{Resultados.}

La figura 1 muestra los la trayectoria cinemática promedio en el plano sagital para las articulaciones de la cadera, rodilla y tobillo inmediatamente antes (curva azul) y después (rojo) de realizar entrenamiento de la marcha durante 15 minutos con el exoesqueleto HANK para las 4 sesiones consideradas. La última fila de la Figura 1 muestra la comparación de los registros previos la tratamiento de las sesiones 1 y 4 . Las principales variables descriptoras, máximo, mínimo, rango de movimiento y valor promedio RMS correspondientes a las gráficas se muestran en las tablas 1 a 3, para las articulaciones de cadera, rodilla y tobillo respectivamente. 
Cambios en la cinemática articular tras entrenamiento de la marcha con exoesqueleto robótico ambulatorio
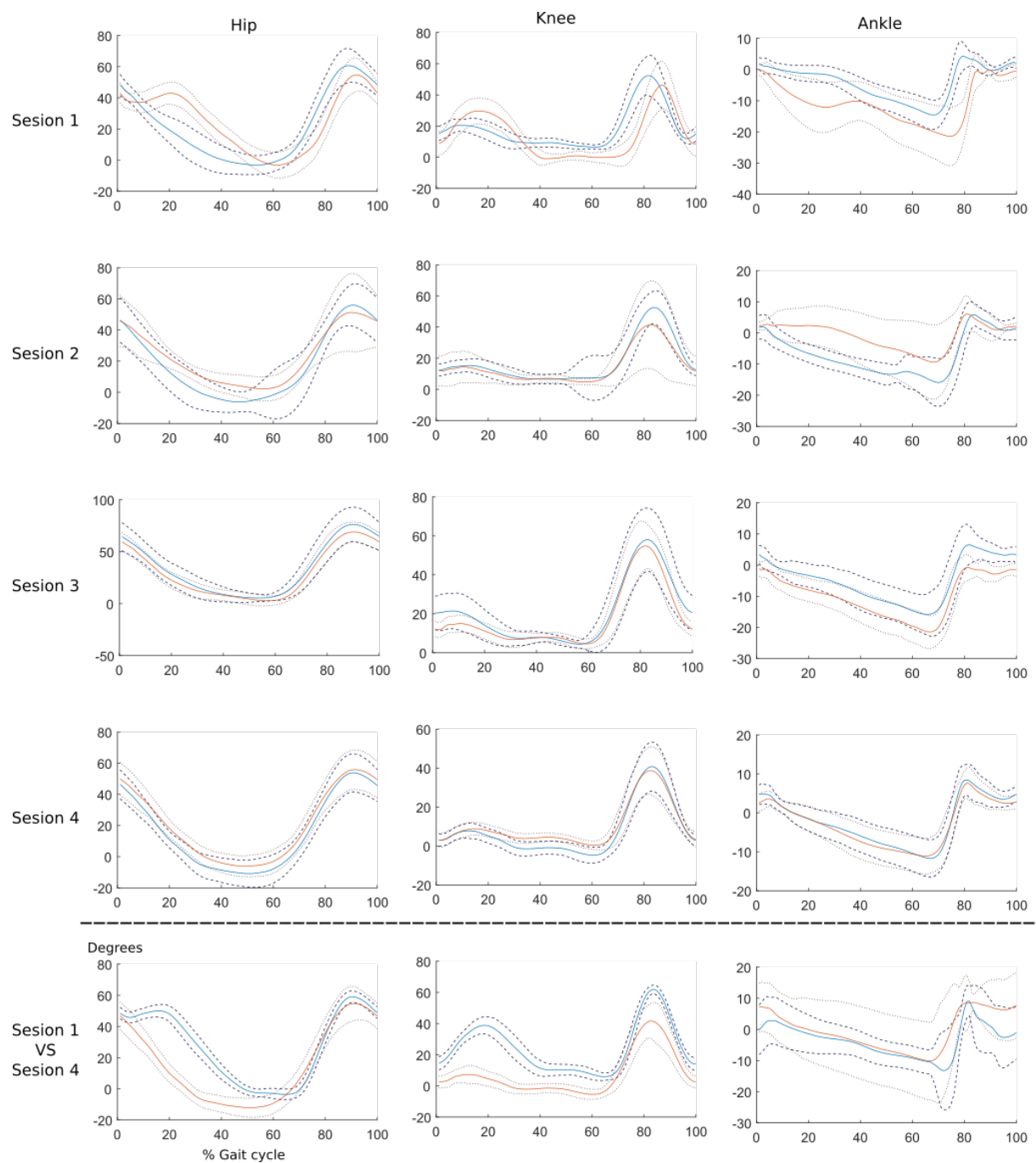

Fig. 1: Angulo de flexión-extensión de las articulaciones de la cadera, rodilla y tobillo (primera, segunda y tercera fila respectivamente) obtenidos inmediatamente antes (curva azul) y después (curva roja) de 15 minutos de entrenamiento de la marcha con el exoesqueleto HANK. La última fila corresponde a la comparación entre las sesiones primera y última de entrenamiento. Las curvas están normalizadas de 0 a $100 \%$ del ciclo de la marcha y representan un ciclo promedio \pm desviación estándar.

La inspección visual de las gráficas muestra diferencias apreciables entre el registro previo y posterior al entrenamiento en la sesión 1, siendo esta diferencia menos apreciable según avanzan las sesiones de tratamiento, llegando a ser casi coincidentes en la sesión. La progresión del paciente en la modificación de las trayectorias articulares se muestra en la 
última fila de la Figura 1, donde se muestra un cambio apreciable en las trayectorias articulares.

Sin embargo, la inspección de los descriptores de las gráficas muestra que siguen existiendo diferencias en los parámetros analizados, por lo que sigue existiendo un efecto del uso del exoesqueleto en el paciente $(\mathrm{p}<0.05)$.

Tabla 1: Parámetros cinemáticos promedio de la flexión-extensión de la cadera. Los datos son media \pm desviación estándar. Los valores en negrita representan significación estadística entre pre- y postentrenamiento para una misma sesión. El * indica significación estadística entre las sesiones 1 y 4.

\begin{tabular}{ll|cccc} 
& & Máximo & Mínimo & $\begin{array}{c}\text { Rango } \\
\text { articular }\end{array}$ & RMS \\
\hline \multirow{2}{*}{ Sesión 1 } & Pre & $59,5 \pm 3.4$ & $\mathbf{- 8 . 6 \pm 3 . 7 *}$ & $\mathbf{6 8 , 1} \pm \mathbf{5 . 4}$ & $\mathbf{3 1 , 0} \pm \mathbf{2 . 9}$ \\
& Post & $58,3 \pm 3.0$ & $\mathbf{- 6 , 9} \pm \mathbf{2 . 8}$ & $\mathbf{6 5 , 1} \pm \mathbf{3 . 2}$ & $\mathbf{3 3 , 3} \pm \mathbf{1 . 9}$ \\
\hline \multirow{2}{*}{ Sesión 2 } & Pre & $\mathbf{5 9 , 9} \pm \mathbf{3 . 1}$ & $\mathbf{- 9 , 4} \pm \mathbf{3 . 9}$ & $69,3 \pm 4.3$ & $\mathbf{3 0 , 5} \pm \mathbf{2 . 6}$ \\
& Post & $\mathbf{6 9 , 2} \pm \mathbf{3 . 0}$ & $\mathbf{- 3 , 1} \pm \mathbf{3 . 6}$ & $72,4 \pm 3.4$ & $\mathbf{3 6 , 1} \pm \mathbf{2 . 2}$ \\
\hline \multirow{2}{*}{ Sesión 3 } & Pre & $\mathbf{8 2 , 7 \pm 3 , 2}$ & $\mathbf{4 , 0} \pm \mathbf{2 , 5}$ & $\mathbf{7 8 , 6} \pm \mathbf{3 , 7}$ & $\mathbf{4 4 , 5} \pm \mathbf{3 , 6}$ \\
& Post & $\mathbf{7 1 , 7} \pm \mathbf{2 , 3}$ & $\mathbf{1 , 7} \pm \mathbf{4 , 0}$ & $\mathbf{7 0 , 0} \pm \mathbf{4 , 2}$ & $\mathbf{3 8 , 7} \pm \mathbf{2 , 1}$ \\
\hline \multirow{2}{*}{ Sesión 4 } & Pre & $57,7 \pm 2,9$ & $\mathbf{- 1 3 , 8} \pm \mathbf{4 , 8 *}$ & $71,5 \pm 4,0^{*}$ & $29,8 \pm 1,9$ \\
& Post & $60,0 \pm 1,6$ & $\mathbf{- 8 , 5} \pm \mathbf{3 , 5}$ & $68,5 \pm 3,2$ & $32,2 \pm 1,3$ \\
\hline
\end{tabular}

Tabla 2: Parámetros cinemáticos promedio de la flexión-extensión de la rodilla. Los datos son media \pm desviación estándar. Los valores en negrita representan significación estadística entre pre- y postentrenamiento para una misma sesión. El * indica significación estadística entre las sesiones 1 y 4.

\begin{tabular}{|c|c|c|c|c|c|}
\hline & & Máximo & Mínimo & $\begin{array}{c}\text { Rango } \\
\text { articular }\end{array}$ & RMS \\
\hline \multirow{2}{*}{ Sesión 1} & Pre & $47,0 \pm 2.1 *$ & $0,9 \pm 2.3^{*}$ & $68,1 \pm 5.4^{*}$ & $18,1 \pm 1.7$ \\
\hline & Post & $52,4 \pm 3.8$ & $-3,5 \pm 1.8$ & $65,1 \pm 3.2$ & $22,4 \pm 2.2$ \\
\hline \multirow{2}{*}{ Sesión 2} & Pre & $57,3 \pm 1.9$ & $1,8 \pm 1.8$ & $69,3 \pm 4.3$ & $23,6 \pm 1.9$ \\
\hline & Post & $62,7 \pm 2.2$ & $4,6 \pm 1.5$ & $72,4 \pm 3.4$ & $27,1 \pm 1.5$ \\
\hline \multirow{2}{*}{ Sesión 3} & Pre & $66,2 \pm 3.0$ & $3,5 \pm 1.2$ & $78,6 \pm 3.7$ & $29,0 \pm 2.5$ \\
\hline & Post & $59,9 \pm 2.8$ & $3,4 \pm 1.6$ & $70,0 \pm 4.2$ & $24,7 \pm 1.4$ \\
\hline \multirow{2}{*}{ Sesión 4} & Pre & $45,3 \pm 1.8 *$ & $-6,3 \pm 2.6 *$ & $71,5 \pm 4.0 *$ & $17,2 \pm 0.9$ \\
\hline & Post & $43,6 \pm 1.9$ & $-1,0 \pm 2.4$ & $68,5 \pm 3.2$ & $16,9 \pm 1.0$ \\
\hline
\end{tabular}


Cambios en la cinemática articular tras entrenamiento de la marcha con exoesqueleto robótico ambulatorio

Tabla 3: Parámetros cinemáticos promedio de la flexión-extensión de la tobillo. Los datos son media \pm desviación estándar. Los valores en negrita representan significación estadística entre pre- y postentrenamiento para una misma sesión. El * indica significación estadística entre las sesiones 1 y 4.

\begin{tabular}{ll|cccc} 
& & Máximo & Mínimo & $\begin{array}{c}\text { Rango } \\
\text { articular }\end{array}$ & RMS \\
\hline \multirow{2}{*}{ Sesión 1 } & Pre & $7,8 \pm 1.0^{*}$ & $\mathbf{- 1 5 , 7 \pm 0 . 9 *}$ & $68,1 \pm 5.4^{*}$ & $\mathbf{8 , 5} \pm \mathbf{1 . 0}$ \\
& Post & $2,7 \pm 1.9$ & $\mathbf{- 2 5 , 1} \pm \mathbf{2 . 6}$ & $65,1 \pm 3.2$ & $\mathbf{1 4 , 4} \pm \mathbf{2 . 0}$ \\
\hline \multirow{2}{*}{ Sesión 2 } & Pre & $\mathbf{8 , 7} \pm \mathbf{1 . 5}$ & $\mathbf{- 1 9 , 8} \pm \mathbf{1 . 7}$ & $\mathbf{6 9 , 3} \pm \mathbf{4 . 3}$ & $\mathbf{1 0 , 2} \pm \mathbf{1 . 3}$ \\
& Post & $\mathbf{1 0 , 7} \pm \mathbf{1 . 0}$ & $\mathbf{- 1 8 , 6} \pm \mathbf{0 . 8}$ & $\mathbf{7 2 , 4} \pm \mathbf{3 . 4}$ & $\mathbf{8 , 6} \pm \mathbf{0 . 5}$ \\
\hline \multirow{2}{*}{ Sesión 3 } & Pre & $\mathbf{1 1 , 3} \pm \mathbf{1 . 0}$ & $\mathbf{- 1 9 , 1} \pm \mathbf{1 . 9}$ & $\mathbf{7 8 , 6} \pm \mathbf{3 . 7}$ & $\mathbf{9 , 9} \pm \mathbf{0 . 9}$ \\
& Post & $\mathbf{1 , 9} \pm \mathbf{2 . 4}$ & $\mathbf{- 2 3 , 3} \pm \mathbf{1 . 8}$ & $\mathbf{7 0 , 0} \pm \mathbf{4 . 2}$ & $\mathbf{1 2 , 7} \pm \mathbf{1 . 1}$ \\
\hline \multirow{2}{*}{ Sesión 4 } & Pre & $11,1 \pm 0.9 *$ & $-13,4 \pm 1.1^{*}$ & $71,5 \pm 4.0^{*}$ & $7,5 \pm 0.5^{*}$ \\
& Post & $9,8 \pm 0.7$ & $-12,6 \pm 1.1$ & $68,5 \pm 3.2$ & $7,5 \pm 0.5$ \\
\hline
\end{tabular}

\section{Discusión.}

La utilización de un exoesqueleto ambulatorio para el entrenamiento de la marcha se fundamenta en los principales principios de la neurorrehabilitación: la realización de tareas funcionales, repetitivas e intensivas, con el objetivo de fomentar los mecanismos neuroplásticos y de aprendizaje que permitan la recuperación de la función alterada.

Hasta la fecha no existen estudios que hayan investigado los cambios cinemáticos inmediatos al entrenamiento con exoesqueleto. Este estudio es el primero que explora los posibles cambios cinemáticos en el plano sagital del entrenamiento de la marcha con exoesqueleto ambulatorio. Los resultados presentados muestran que efectivamente parece que existe un cierto efecto posterior al entrenamiento, que parece instaurarse en el paciente a través de las 4 sesiones analizadas. La inspección de las curvas muestra una adaptación progresiva hacia los patrones finales, similares a los patrones cinemáticos impuestos por el exoesqueleto, que provienen a su vez de una base de datos normativa [28]. No obstante, el análisis estadístico realizado a los descriptores de las curvas muestra diferencias significativas en todas las sesiones, incluso en la sesión 4, donde las curvas aparentemente muestran una gran igualdad.

Este trabajo abre numerosas cuestiones importantes para el campo de la neurorrehabilitación de la marcha con robots de asistencia. En primer lugar, es necesario estudiar si existen cambios en las articulaciones en los planos frontal y coronal como consecuencia de la restricción de movimiento del exoesqueleto. En este trabajo se ha 
obviado este análisis debido a la extensión del mismo, y porque a priori no cabe esperar demasiados cambios en estos grados de libertad dado que no son movilizados por el robot.

El efecto de la intensidad de la terapia en el efecto tras entrenamiento debe ser estudiado. La pregunta subyacente es si existe una dosis o tiempo de entrenamiento por encima del cual no se producen cambios en la cinemática articular, siendo entonces innecesario continuar el entrenamiento en esa sesión.

Este estudio cuenta con varias limitaciones. En primer lugar, el bajo número de sesiones de entrenamiento analizado, así como las características particulares de la paciente impide obtener conclusiones generalizables sobre el proceso de aprendizaje. Además, la adquisición de la cinemática mediante los sensores inerciales presenta limitaciones vinculadas a las prestaciones de esta tecnología y el procedimiento de registro. La tecnología de medida ambulatoria inercial presenta limitaciones relacionadas con la deriva de los sensores y las perturbaciones electromagnéticas que limitan la precisión de las muestras obtenidas. Por otra parte, la calibración anatómica de la orientación de los sensores a la orientación de los segmentos introduce también alteraciones en las medidas.

\section{Conclusión.}

El presente estudio ha mostrado que existen cambios en la cinemática articular durante las sesiones de entrenamiento de la marcha con exoesqueleto. Los cambios tienden a reducirse según aumentan las sesiones de entrenamiento, aunque no se ha podido demostrar estadísticamente. El procedimiento experimental puede considerarse válido, siendo este estudio la base para un análisis más amplio con un mayor número de sujetos y sesiones de entrenamiento.

\section{Agradecimientos.}

Este trabajo ha sido posible gracias a los proyectos Bidirectional Hyper-Connected Neural System (EXTEND), del programa de investigación e innovación H2020, (Ref. 779982), y Sistemas Modulares Robóticos y Neuroprotésicos Personalizables para la Asistencia de la Marcha Patológica (TAILOR), financiado por el Ministerio de Ciencia, Innovación y Universidades, dentro del programa de Proyectos de I+D+i Retos de Investigación (Ref. RTI2018-097290-B-C31) 


\section{Referencias.}

[1] M. Wyndaele and J. J. Wyndaele, "Incidence, prevalence and epidemiology of spinal cord injury: What learns a worldwide literature survey?," Spinal Cord, vol. 44, no. 9, pp. 523-529, 2006.

[2] P. L. Ditunno, M. Patrick, M. Stineman, and J. F. Ditunno, "Who wants to walk? Preferences for recovery after SCI: A longitudinal and cross-sectional study," Spinal Cord, vol. 46, no. 7, pp. 500-506, 2008.

[3] G. Colombo, M. Wirz, and V. Dietz, "Driven gait orthosis for improvement of locomotor training in paraplegic patients," in Spinal Cord, 2001, vol. 39, no. 5, pp. 252-255.

[4] G. Colombo, M. Joerg, R. Schreier, and V. Dietz, "Treadmill training of paraplegic patients using a robotic orthosis," J. Rehabil. Res. Dev., vol. 37, no. 6, pp. 693-700, 2000.

[5] S. Freivogel, J. Mehrholz, T. Husak-Sotomayor, and D. Schmalohr, "Gait training with the newly developed'LokoHelp'-system is feasible for non-ambulatory patients after stroke, spinal cord and brain injury. A feasibility study," Brain Inj., vol. 22, no. 7-8, pp. 625-632, 2008.

[6] S. Hesse, A. Waldner, and C. Tomelleri, "Innovative gait robot for the repetitive practice of floor walking and stair climbing up and down in stroke patients," J. Neuroeng. Rehabil., vol. 7, no. 1, p. 30, Jan. 2010.

[7] C. Nooijen, N. Ter Hoeve, and E. Field-Fote, "Gait quality is improved by locomotor training in individuals with SCI regardless of training approach," J. Neuroeng. Rehabil., vol. 6, no. 1, p. 36, Jan. 2009.

[8] C. Morawietz and F. Moffat, "Effects of locomotor training after incomplete spinal cord injury: A systematic review," Arch. Phys. Med. Rehabil., vol. 94, no. 11, pp. 2297-2308, 2013.

[9] M. Wessels, C. Lucas, I. Eriks-Hoogland, and S. De Groot, "Body weight-supported gait training for restoration of walking in people with an incomplete spinal cord injury: A systematic review," Assist. technol. Res. Ser., vol. 26, no. 6, pp. 297-299, Jun. 2010.

[10] E. Swinnen, S. Duerinck, J. Baeyens, R. Meeusen, and E. Kerckhofs, "Effectiveness of robotassisted gait training in persons with spinal cord injury: A systematic review," J. Rehabil. Med., vol. 42, no. 6, pp. 520-526, Jun. 2010.

[11] J. Mehrholz, J. Kugler, and M. Pohl, "Locomotor training for walking after spinal cord injury," Cochrane database Syst. Rev., vol. 11, no. 11, p. CD006676, 2012.

[12] B. H. Dobkin and P. W. Duncan, "Should body weight-supported treadmill training and roboticassistive steppers for locomotor training trot back to the starting gate?," Neurorehabil. Neural Repair, vol. 26, no. 4, pp. 308-317, May 2012.

[13] A. Wall, J. Borg, and S. Palmcrantz, "Clinical application of the Hybrid Assistive Limb (HAL) for gait training: a systematic review," Front. Syst. Neurosci., vol. 9, no. 9, pp. 483389-48, 2015.

[14] M. Bortole et al., "The H2 robotic exoskeleton for gait rehabilitation after stroke: Early findings from a clinical study Wearable robotics in clinical testing," J. Neuroeng. Rehabil., vol. 12, no. 1, pp. 1-14, 2015.

[15] V. Lajeunesse, C. Vincent, F. Routhier, E. Careau, and F. Michaud, "Exoskeletons' design and usefulness evidence according to a systematic review of lower limb exoskeletons used for 
functional mobility by people with spinal cord injury," Disabil. Rehabil. Assist. Technol., vol. 11, no. 7, pp. 535-547, 2016.

[16] S. Federici, F. Meloni, M. Bracalenti, and M. L. De Filippis, "The effectiveness of powered, active lower limb exoskeletons in neurorehabilitation: A systematic review," NeuroRehabilitation, vol. 37, no. 3, pp. 321-340, 2015.

[17] J. L. Contreras-Vidal et al., "Powered exoskeletons for bipedal locomotion after spinal cord injury," J. Neural Eng., vol. 13, no. 3, pp. 1-16, 2016.

[18] D. R. Louie, J. J. Eng, and T. Lam, "Gait speed using powered robotic exoskeletons after spinal cord injury: A systematic review and correlational study," J. Neuroeng. Rehabil., vol. 12, no. 1, p. 82, 2015.

[19] S. J. Harkema, J. Hillyer, M. Schmidt-Read, E. Ardolino, S. A. Sisto, and A. L. Behrman, "Locomotor Training: As a treatment of spinal cord injury and in the progression of neurologic rehabilitation," Arch. Phys. Med. Rehabil., vol. 93, no. 9, pp. 1588-1597, 2012.

[20] V. Dietz, "Body weight supported gait training: From laboratory to clinical setting," Brain Research Bulletin, vol. 76, no. 5. pp. 459-463, 2008.

[21] S. Jezernik, G. Colombo, T. Keller, H. Frueh, and M. Morari, "Robotic Orthosis Lokomat: A Rehabilitation and Research Tool," Neuromodulation, vol. 6, no. 2, pp. 108-115, 2003.

[22] N. Evans, C. Hartigan, C. Kandilakis, E. Pharo, and I. Clesson, "Acute Cardiorespiratory and Metabolic Responses During Exoskeleton-Assisted Walking Overground Among Persons with Chronic Spinal Cord Injury,” Top. Spinal Cord Inj. Rehabil., vol. 21, no. 2, pp. 122-132, 2015.

[23] N. Lefeber, E. Swinnen, M. Michielsen, S. Henderix, and E. Kerckhofs, "Energy consumption and cardiorespiratory load during lokomat walking compared to walking without robotassistance in stroke patients: Preliminary results," in Biosystems and Biorobotics, vol. 15, J. Ibáñez, J. González-Vargas, J. M. Azorín, M. Akay, and J. L. Pons, Eds. Cham: Springer International Publishing, 2017, pp. 1229-1233.

[24] A. Esquenazi, M. Talaty, and A. Jayaraman, "Powered Exoskeletons for Walking Assistance in Persons with Central Nervous System Injuries: A Narrative Review," $P M R$, vol. 9, no. 1, pp. 46-62, Jan. 2017.

[25] L. J. Holanda, P. M. M. Silva, T. C. Amorim, M. O. Lacerda, C. R. Simão, and E. Morya, "Robotic assisted gait as a tool for rehabilitation of individuals with spinal cord injury: a systematic review," J. Neuroeng. Rehabil., vol. 14, no. 1, p. 126, 2017.

[26] E. Y. Y. Cheung, T. K. W. Ng, K. K. K. Yu, R. L. C. Kwan, and G. L. Y. Cheing, "RobotAssisted Training for People With Spinal Cord Injury: A Meta-Analysis," Arch. Phys. Med. Rehabil., vol. 98, no. 11, pp. 2320-2331.e12, 2017.

[27] F. Sylos-Labini et al., "EMG patterns during assisted walking in the exoskeleton," Front. Hum. Neurosci., vol. 8, no. June, p. 423, 2014.

[28] C. A. Fukuchi, R. K. Fukuchi, and M. Duarte, "A public dataset of overground and treadmill walking kinematics and kinetics in healthy individuals," PeerJ, vol. 6, p. e4640, 2018. 